\title{
BMJ Open Time-varying impact of comorbidities on mortality after liver transplantation: a national cohort study using linked clinical and administrative data
}

\author{
Chutwichai Tovikkai, ${ }^{1,2}$ Susan C Charman, ${ }^{1,3}$ Raaj K Praseedom, ${ }^{2,4}$ \\ Alexander E Gimson, ${ }^{4}$ Jan van der Meulen ${ }^{1,3}$
}

To cite: Tovikkai C, Charman SC, Praseedom RK, et al. Time-varying impact of comorbidities on mortality after liver transplantation: a national cohort study using linked clinical and administrative data. BMJ Open 2015;5:e006971. doi:10.1136/bmjopen-2014006971

- Prepublication history for this paper is available online. To view these files please visit the journal online (http://dx.doi.org/10.1136/ bmjopen-2014-006971).

Received 21 October 2014 Revised 20 January 2015 Accepted 6 March 2015

\section{CrossMark}

\footnotetext{
${ }^{1}$ Clinical Effectiveness Unit, The Royal College of Surgeons of England, London, UK

${ }^{2}$ Department of Surgery, University of Cambridge, Cambridge, UK

${ }^{3}$ Department of Health Services Research and Policy, London School of Hygiene and Tropical Medicine, London, UK

${ }^{4}$ Liver Transplant Unit, Addenbrooke's Hospital, Cambridge University Hospitals NHS Foundation Trust, Cambridge, UK
}

Correspondence to Dr Chutwichai Tovikkai; ct422@cam.ac.uk

\section{ABSTRACT}

Objective: We assessed the impact of comorbidity on mortality in three periods after liver transplantation (first 90 days, 90 days -5 years and $5-10$ years).

Design: Prospective cohort study using records from the UK Liver Transplant Audit (UKLTA) linked to Hospital Episode Statistics (HES), an administrative database of hospital admissions in the English National Health Service (NHS). Comorbidities relevant for liver transplantation were identified from the 10th revision of the International Classification of Diseases (ICD-10) codes in HES records of admissions in the year preceding their operation. Multivariable Cox regression was used to estimate HRs for three different time periods after liver transplantation.

Setting: All liver transplant centres in the NHS hospitals in England.

Participants: Adults who received a first elective liver transplant between April 1997 and March 2010 in the linked UKLTA-HES database.

Outcomes: Patient mortality in three different time periods after transplantation.

Results: Among 3837 recipients, $45.1 \%$ had comorbidities. Recipients with cardiovascular disease had statistically significantly higher mortality in all three periods after transplantation (first 90 days: $\mathrm{HR}=2.0 ; 95 \%$ $\mathrm{Cl} 1.4$ to $2.9,90$ days -5 years: $1.6 ; 1.2$ to 2.2 , beyond 5 years: $2.8 ; 1.7$ to 4.4 ). Prior congestive cardiac failure (3.2; 2.1 to 4.9$)$ significantly increased mortality only in the first 90 days. History of non-hepatic malignancy appeared to increase risk over all periods, but significantly only in the first 90 days $(1.9 ; 1.0$ to 3.6$)$. A diagnosis of connective tissue disease, dementia, diabetes, chronic pulmonary and renal disease did not have a significant impact on mortality in any period.

Conclusions: The impact of comorbidities present at the time of transplantation changes with time after transplantation. Renal disease, pulmonary disease and diabetes had no impact on mortality in contrast to previous reports.

\section{INTRODUCTION}

Comorbidity is an important determinant of outcome in medical and surgical patients. ${ }^{1-6}$

\section{Strengths and limitations of this study}

- A large national clinical database with linkage to administrative data was used, and a newly developed instrument to capture comorbidity information relevant to liver transplant patients from administrative data was applied.

- Multivariable Cox regression analysis including interaction between comorbidities and time periods was used to show that some comorbidities had a different impact on survival in different time periods after liver transplantation.

- Our comorbidity instrument has been adapted from the Charlson Comorbidity Index, which captures important comorbidities. However, some minor health problems may not have been included.

However, in liver transplant patients, the reported impact of comorbidities on the outcome varies widely. ${ }^{7-11}$ One frequently used tool to measure comorbid conditions is the Charlson Comorbidity Index. ${ }^{12}$ Singlecentre studies from the USA ${ }^{13}$ and Italy ${ }^{14}$ have demonstrated that this index is associated with outcome after liver transplantation. Nevertheless, other studies suggest that it does not reliably predict short-term posttransplant mortality. ${ }^{15} 16$

Comorbidities may have a different impact on mortality in different time periods after liver transplantation. ${ }^{17}{ }^{18}$ For example, congestive cardiac failure may have an impact on short-term mortality ${ }^{19}$ but not in the long term. Diabetes mellitus may gradually damage tissues and thus have an impact on mortality in the long term. ${ }^{8}$

We investigated the impact of comorbidities on patient mortality in three time periods after liver transplantation (first 90 days, 90 days -5 years and $5-10$ years). To do this, we linked the UK Liver Transplant 
Audit (UKLTA) database at patient level to the Hospital Episode Statistics (HES), an administrative database of all admissions to English National Health Service (NHS) hospitals. A specific coding scheme was developed to identify comorbidities relevant for liver transplantation from the HES diagnosis codes, which are based on the 10th revision of the International Classification of Diseases (ICD-10). This coding scheme is an adaptation of the Royal College of Surgeons (RCS) Charlson Score, which has been validated in various groups of surgical patients. ${ }^{20-23}$

\section{MATERIALS AND METHODS}

The adaptation of the RCS Charlson Score for use in liver transplantation

The RCS Charlson Score is an ICD-10-based comorbidity score that can be derived from administrative hospital records and is developed for use in surgical patients. We adapted this score to make it suitable for patients receiving a liver transplant by following a number of coding principles:

1. A morbidity that is linked to an indication for liver transplantation, even it affects other organs, should not be coded as a comorbidity.

2. A morbidity that can be caused by liver disease should not be coded as a comorbidity.

3. Comorbidities that are parts of the same spectrum of disease should be grouped together.

4. A comorbidity with very low prevalence (ie, less than $1 \%$ ) should be ignored.

Comorbidities were identified from ICD-10 codes in HES records. These codes were sought from the index admission (the admission for liver transplantation) and also from all admissions in the year before the transplant. Some ICD-10 codes that reflect acute conditions such as acute renal failure (marked with $*$ in table 1 ) were defined as comorbidities only if they were present in preceding admissions. If these codes were present only in the index admission, they were ignored as it is uncertain whether they are truly comorbidities or complications that occurred perioperatively. ${ }^{20}$

Conditions linked to indications for liver transplantation (eg, chronic liver disease) were not considered as comorbidities. Also, we excluded codes for particular cancers that can lead to indications for liver transplantation: C22 (primary liver cancer), C24 (cancer of biliary tract) and C25 (endocrine pancreatic cancer) or C75.9 (other endocrine cancer) with C78.7 (liver metastasis of neuroendocrine cancer). The code C77 (lymph node metastasis) was also excluded as it reflects a staging of malignant disease rather than a diagnosis itself. Codes for other metastatic solid tumours were combined and considered as a single comorbidity.

Myocardial infarction, peripheral vascular disease, cerebrovascular disease were grouped together as cardiovascular disease because they all have the physiological impact on the cardiovascular system of a patient. Congestive cardiac failure was included as a separate group because it can also result from other causes, for example, valvular heart disease, non-ischaemic cardiomyopathy. Hemiplegia/paraplegia and HIV/AIDS had a very low prevalence in our cohort of liver transplant recipients $(0.2 \%$ and $0.3 \%$, respectively), and were therefore ignored. Constructing the adapted Charlson score using the above principles resulted in eight comorbidity categories (table 1).

\section{The linked UKLTA-HES database}

We used records from UKLTA linked at patient level to records from HES. The UKLTA database prospectively collects pretransplant recipient, donor, perioperative and follow-up data including survival information for all patients undergoing liver transplantation in the UK for audit purposes. ${ }^{24}$ The HES database contains administrative records of all admissions to NHS hospitals in

Table 1 Eight comorbidities from the Royal College of Surgeons Charlson Score adapted for use in liver transplant patients and their ICD-10 codes

\begin{tabular}{|c|c|}
\hline Disease category & ICD-10 codes \\
\hline \multicolumn{2}{|l|}{ 1. Cardiovascular disease } \\
\hline 1.1 Myocardial infarction & $121^{*}, 122^{*}, 123^{*}, 125.2$ \\
\hline 1.2 Peripheral vascular disease & I70-I73, I77.0, I77.1, K55.1, K55.8, K55.9, R02, Z95.8, Z95.9 \\
\hline 1.3 Cerebrovascular disease & G45, G46, I60-169 \\
\hline 2. Congestive cardiac failure & $111,|13| 25.5,142,, \mid 43,150,151.7$ \\
\hline 3. Connective tissue disease & M05, M06, M09, M12.0, M31.5, M32-M36 \\
\hline 4. Dementia & A81.0, F00-F03, F05.1, G30, G31 \\
\hline 5. Diabetes mellitus & E10-E14 \\
\hline 6. Non-hepatic malignancy & $\begin{array}{l}\text { C00-C21, C26, C30-C34, C37-C41, C43, C45-C58, C60-C74, } \\
\text { C75.0-C75.8, C76, C78.0-C78.6, C78.8, C79, C80-C85, C88, C90-C97 }\end{array}$ \\
\hline 7. Chronic pulmonary disease & I26, I27, J40-J45, J46*, J47, J60-J67, J68.4, J70.1, J70.3 \\
\hline 8. Chronic renal disease & I12, I13, N01, N03, N05, N07, N08, N17.1* , N17.2*, N18, N19*, N25, Z49, Z94.0, Z99.2 \\
\hline
\end{tabular}


England. $^{25}$ HES records contain ICD-10 diagnosis $\operatorname{codes}^{26}$ and procedure codes based on the fourth revision of the Office for Population Censuses and Survey classification of interventions and procedures (OPCS-4) codes. ${ }^{27}$ The linkage was based on hierarchical deterministic linkage criteria, including NHS number, sex, date of birth, postcode, date of transplantation and a procedure code for liver transplantation or a diagnosis code relevant to liver disease. This linked data set contained records of patients receiving a first liver transplant in the UK between 1 April 1997 and 31 March 2010. A detailed description and an evaluation of the linkage process have been published elsewhere. ${ }^{28}$ All patients were followed up for at least 2 years.

We excluded linked records of paediatric liver transplantation (ie, recipients younger than 17 years), multiorgan transplantation, living-donor liver transplantation, domino liver transplantation and super urgent liver transplantation.

\section{Statistical analysis}

The prevalence of each individual comorbidity and number of concomitant comorbidities in each patient were described according to the era of transplantation. Unadjusted mortality rates were calculated using the Kaplan-Meier method and described as percentages with $95 \%$ CIs. The impact of comorbidities on mortality after liver transplantation was assessed with multivariable Cox regression, allowing for three time periods after liver transplantation: the first 90 days, 90 days -5 years and beyond 5 years. These cut-off points are commonly used in the UK to describe short-term and mid-term transplant outcome..$^{29}$ Mortality in the first 90 -day period mostly reflects surgical outcome including complications from liver transplant operations, while mortality after 90 days is probably caused by long-term medical complications including those related to immunosuppression. Furthermore, in the UK, a patient is accepted on to the transplant list if the potential recipient is predicted to have at least $50 \%$ 5-year post-transplant survival. ${ }^{30}$ The analysis was censored at 10 years after transplantation.

Results are presented as HRs and 95\% CIs, separately for each of the three time periods. To assess the change in HRs over the three time periods, we conducted an interaction test between time period and comorbidity. A $\mathrm{p}$ value less than 0.05 was considered to indicate that the effect of the comorbidity significantly changes over time.

The multivariable model included all comorbidities, age, sex, the primary liver disease (9 groups), era of liver transplantation (categorised into 4 eras), pretransplant mechanical ventilation, previous abdominal surgery, serum creatinine, bilirubin and sodium, donor type (donor after brain death vs donor after cardiac death), graft appearance (healthy vs suboptimal) and graft type (whole vs segmental). All factors were analysed as binary or continuous variables unless otherwise stated. A goodness-of-fit test was performed to confirm that the multivariable model is well calibrated and fits the data well by comparing the observed mortality with the expected mortality as predicted by the model in 10 equally sized groups ranked according to the model's predicted mortality. ${ }^{31}$

We used multiple imputation by chained equations ${ }^{32}$ to deal with any missing values of risk factors in UKLTA data. Missing values were replaced with 10 sets of plausible values. These 10 data sets were analysed individually and the estimates were then combined to obtain the overall estimates by using Rubin's rules and rules for combining $\chi^{2}$ statistics. ${ }^{33}$ No variable in the model had more than $10 \%$ missing values. Stata V.11.2 (StataCorp, College Station, Texas, USA) was used for all statistical analyses.

\section{RESULTS}

\section{Patient and perioperative characteristics}

The linked UKLTA-HES data set of adult elective first liver transplants in our study consisted of 3837 patients. Median and IQR of age were 53 (46-60) years, and 63\% of the recipients were male. The most common primary liver disease was alcoholic liver disease (22.5\%), while $17.3 \%$ had cancer as an indication for transplantation (table 2). Median (IQR) follow-up time was 4.7 (1.9-8.0) years with range from 0 to 14.1 years.

\section{Prevalence of the comorbidities}

Diabetes mellitus was the most common comorbidity with a prevalence of $23.9 \%$. Chronic pulmonary disease $(9.9 \%)$ was the second and chronic renal disease $(7.7 \%)$ the third most common comorbidity (table 3 ).

Overall, $45.1 \%$ of the patients had at least one comorbidity. About one-third had one comorbidity, $10 \%$ had two comorbidities and less than $3 \%$ had three comorbidities or more (table 3). The most common combinations of comorbidities among patients who had two comorbidities were diabetes with chronic pulmonary disease (69 patients), and diabetes with chronic renal disease (67 patients). Diabetes with renal disease and cardiovascular disease (12 patients), and diabetes with pulmonary disease and cardiovascular disease (10 patients) were the most common combinations among patients with three comorbidities.

The prevalence of some comorbidities changed according to the era of transplantation. Diabetes was found in $17.4 \%$ of liver transplant patients during 1997 2000, and this increased to 30\% between 2006 and 2010 . Chronic renal disease had an increased prevalence from $4.3 \%$ to $10.9 \%$ during the same period. The prevalence of chronic pulmonary disease and dementia also increased during these eras. The prevalence of cardiovascular disease, congestive cardiac failure and connective tissue disease remained stable, while the prevalence of a history of non-hepatic malignancy decreased over time. The number of patients with more than one comorbidity also increased with time. Only $0.9 \%$ of the patients transplanted between 1997 and 2000 had three or more comorbidities, whereas this increased to $5.1 \%$ in those transplanted between 2006 and 2010 (table 3). 
Table 2 Characteristics of the adult recipients of an elective first liver transplant in the linked UK Liver Transplant Audit database and Hospital Episode Statistics

\begin{tabular}{|c|c|c|}
\hline Characteristic & Value & Missing (N) \\
\hline Number & 3837 & \\
\hline Age (year) & $53(46-60)$ & 0 \\
\hline $\operatorname{Sex}(\%)$ & & 0 \\
\hline Male & $2400(62.6)$ & \\
\hline Female & $1437(37.5)$ & \\
\hline Ethnicity (\%) & & 0 \\
\hline White & 3350 (87.3) & \\
\hline Asian & $361(9.4)$ & \\
\hline Black & $89(2.3)$ & \\
\hline Other & $37(1.0)$ & \\
\hline BMI $\left(\mathrm{kg} / \mathrm{m}^{2}\right)$ & $25.6(22.7-29.1)$ & 215 \\
\hline Primary liver disease (\%) & & 0 \\
\hline Cancer & $662(17.3)$ & \\
\hline Hepatitis C cirrhosis & $462(12.0)$ & \\
\hline Primary sclerosing cholangitis & $410(10.7)$ & \\
\hline Hepatitis B cirrhosis & $107(2.8)$ & \\
\hline Primary biliary cirrhosis & $576(15.0)$ & \\
\hline Alcoholic cirrhosis & $867(22.5)$ & \\
\hline Autoimmune and cryptogenic cirrhosis & $391(10.2)$ & \\
\hline Metabolic liver disease & $132(3.4)$ & \\
\hline Other liver disease & $233(6.1)$ & \\
\hline Era of liver transplantation (\%) & & 0 \\
\hline April 1997-September 2000 & $922(24.0)$ & \\
\hline October 2000-September 2003 & $882(23.0)$ & \\
\hline October 2003-September 2006 & 915 (23.9) & \\
\hline October 2006-March 2010 & $1118(29.1)$ & \\
\hline Pretransplant mechanical ventilation (\%) & $23(0.6)$ & 1 \\
\hline Previous abdominal surgery (\%) & $598(15.6)$ & 7 \\
\hline Bilirubin $(\mu \mathrm{mol} / \mathrm{L})$ & 46 (24-95) & 19 \\
\hline Creatinine $(\mu \mathrm{mol} / \mathrm{L})$ & $87(73-104)$ & 2 \\
\hline INR & $1.3(1.2-1.6)$ & 145 \\
\hline Sodium (mmol/L) & $137(134-139)$ & 9 \\
\hline Donor type (\%) & & 0 \\
\hline Donor after brain death & 3624 (94.4) & \\
\hline Donor after cardiac death & $213(5.6)$ & \\
\hline Graft type (\%) & & 0 \\
\hline Whole & 3568 (93.0) & \\
\hline Segmental & $269(7.0)$ & \\
\hline Graft appearance (\%) & & 313 \\
\hline Healthy & $2834(80.4)$ & \\
\hline Suboptimal & $690(19.6)$ & \\
\hline
\end{tabular}

Effects of comorbidities on post-transplant mortality

Overall, the 90-day mortality rate was $7.0 \%(95 \%$ CI $6.3 \%$ to $7.9 \%$ ). The 5 -year mortality rate was $24.1 \%$ $(22.6 \%$ to $25.6 \%)$, and the 10 -year mortality rate was $37.6 \%(35.5 \%$ to $39.7 \%)$.

Patients with congestive cardiac failure had the highest 90-day mortality rate at $20.2 \%$ (95\% CI $13.9 \%$ to $28.8 \%$; figure 1). Patients with a history of non-hepatic malignancy $(15.6 \% ; 8.7 \%$ to $27.1 \%)$, cardiovascular disease $(12.4 \% ; 8.9 \%$ to $17.2 \%)$ and chronic renal disease $(9.9 \%$; $7.0 \%$ to $13.9 \%$ ) also had an increased 90-day mortality. The highest 5-year mortality rates were found in recipients with a history of non-hepatic malignancy $(39.9 \% ; 28.6 \%$ to $53.6 \%)$, congestive cardiac failure $(37.5 \% ; 28.2 \%$ to $48.7 \%)$, cardiovascular disease $(35.5 \% ; 29.1 \%$ to $42.8 \%)$ and chronic renal disease $(31.2 \% ; 25.5 \%$ to $37.8 \%)$. Patients with cardiovascular disease had the highest 10 -year mortality rate at $60.0 \%$ ( $50.3 \%$ to $70.0 \%)$.

Effects of comorbidities on mortality in different time periods after liver transplantation

Cardiovascular disease was associated with a statistically significantly increased risk of mortality in all three time periods after liver transplantation. The change in the risk 
Table 3 Prevalence (\%) of each individual comorbidity and distribution (\%) of number of comorbidities in liver transplant patients categorised by era of transplantation

\begin{tabular}{|c|c|c|c|c|c|c|}
\hline \multirow[b]{2}{*}{ Comorbidity } & \multicolumn{4}{|c|}{ Era of transplantation } & \multicolumn{2}{|l|}{ Overall } \\
\hline & 1997-2000 & $2000-2003$ & 2003-2006 & 2006-2010 & Prevalence (\%) & $\mathbf{N}$ \\
\hline $\mathrm{N}$ & 922 & 882 & 915 & 1118 & & 3837 \\
\hline Cardiovascular disease & 6.0 & 5.1 & 6.2 & 8.3 & 6.5 & 250 \\
\hline Myocardial infarction & 0.8 & 0.6 & 1.3 & 1.2 & 1.0 & 37 \\
\hline Peripheral vascular disease & 3.0 & 3.6 & 3.3 & 4.9 & 3.8 & 145 \\
\hline Cerebrovascular disease & 2.3 & 1.0 & 1.9 & 2.4 & 1.9 & 74 \\
\hline Congestive cardiac failure & 2.5 & 2.2 & 3.0 & 4.0 & 3.0 & 114 \\
\hline Connective tissue disease & 3.7 & 3.4 & 4.5 & 3.4 & 3.7 & 143 \\
\hline Dementia & 2.3 & 3.5 & 5.9 & 7.3 & 4.9 & 187 \\
\hline Diabetes mellitus & 17.4 & 21.9 & 25.0 & 30.0 & 23.9 & 917 \\
\hline Non-hepatic malignancy & 2.4 & 1.8 & 1.4 & 1.2 & 1.7 & 64 \\
\hline Chronic pulmonary disease & 6.5 & 6.9 & 11.3 & 13.8 & 9.9 & 378 \\
\hline Chronic renal disease & 4.3 & 6.0 & 9.4 & 10.9 & 7.7 & 294 \\
\hline \multicolumn{7}{|l|}{ Number of comorbidities } \\
\hline 0 & 62.6 & 59.8 & 52.2 & 46.8 & 54.9 & 2105 \\
\hline 1 & 30.7 & 30.3 & 32.5 & 34.4 & 32.1 & 1230 \\
\hline 2 & 5.9 & 9.4 & 12.0 & 13.8 & 10.5 & 402 \\
\hline 3 or more & 0.9 & 0.6 & 3.3 & 5.1 & 2.5 & 100 \\
\hline
\end{tabular}

of mortality across the three time periods after transplantation was not statistically significant (interaction test: $\mathrm{p}=0.14)$. Nevertheless, the risk was the highest after 5 years with an adjusted HR of 2.8 (95\% CI 1.7 to 4.4 ). The adjusted HRs were 2.0 (1.4 to 2.9) for the first 90 days and 1.6 (1.2 to 2.2 ) for 90 days-5 years (figure 2 ).

Patients with congestive cardiac failure had a significantly higher 90-day mortality risk than those without (adjusted HR=3.2; 95\% CI 2.1 to 4.9). However, this excess risk was not observed beyond 90 days (90 days5 years: $1.9 ; 0.7$ to 2.0 , beyond 5 years: $0.8 ; 0.3$ to 2.6 ; figure 2). This observed reduction of risk across the three time periods was statistically significant (interaction test: $\mathrm{p}=0.01$ ).

In all three time periods, a history of non-hepatic malignancy was associated with a similar increase in the risk of death (interaction test: $\mathrm{p}=0.84$ ). However, this was statistically significant only in the first 90 days (adjusted $\mathrm{HR}=1.9$; $95 \%$ CI 1.0 to 3.6). The corresponding figures for the periods of 90 days -5 years and beyond 5 years after transplantation were 1.6 (0.9 to 2.7) and 2.0 (0.9 to 4.5 ), respectively (figure 2 ).

The remaining five comorbidities, connective tissue disease, dementia, diabetes, chronic pulmonary disease and chronic renal disease, did not show a statistically significant association with mortality in any of the time periods. However, there was some evidence to suggest that chronic renal disease increased the risk of death in the first 90 days (adjusted HR=1.4; 95\% CI 0.9 to 2.1), and the mortality of patients with dementia increased beyond 5 years $(1.8 ; 0.9$ to 3.4$)$. The interaction test for diabetes $(\mathrm{p}=0.09)$ indicates that the effect of diabetes on mortality may change over the follow-up time, whereas the changes in the HRs across time periods for other comorbidities were not significant (figure 2).
The goodness-of-fit test of the multivariable Cox regression model showed no significant difference between expected and observed mortality in 10 equally divided groups, and suggested that the model was well calibrated $(\mathrm{p}=0.69)$.

\section{DISCUSSION}

This study demonstrates that some comorbidities diagnosed before transplantation have a significant impact on post-transplant mortality and that the impact may vary according to time after transplantation. Cardiovascular disease increases the risk of post-transplant mortality in all time periods up to 10 years. In contrast, we observed an increased risk only in the short term for patients with congestive cardiac failure. A history of non-hepatic malignancy seems to be associated with higher mortality for the whole follow-up period. This detailed analysis of the impact of comorbidity was only possible because we developed a specific coding scheme to extract information on comorbidities relevant for liver transplantation from ICD-10 diagnosis codes in linked administrative hospital records for a large number of liver transplant patients.

Diabetes mellitus was the most common comorbidity (24\%), similar to studies in the $\mathrm{USA}^{13}$ and Italy. ${ }^{14}$ Chronic pulmonary disease $(10 \%)$ and chronic renal disease $(8 \%)$ were found to be the second and third most common comorbidities, which corresponds to their prevalence reported in the Italian study ( $11 \%$ for pulmonary disease and $7 \%$ for renal disease).

Nevertheless, the prevalence of some comorbidities was clearly different from other studies. This may result from variations in the definition of comorbidities. For example, the prevalence of chronic renal disease is $16 \%$ in the US study, which used a serum creatinine level of 
Cardiovascular disease

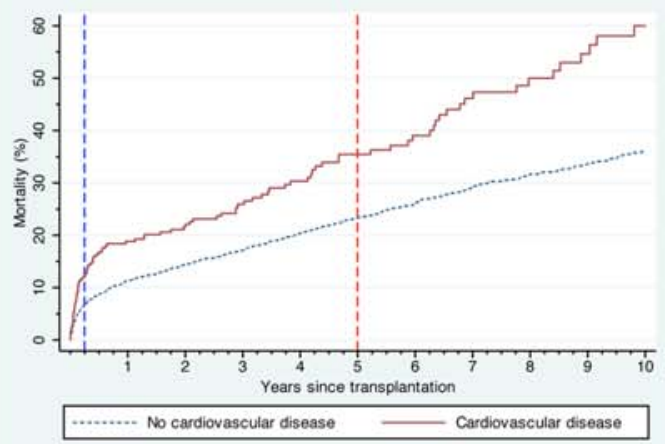

Connective tissue disease

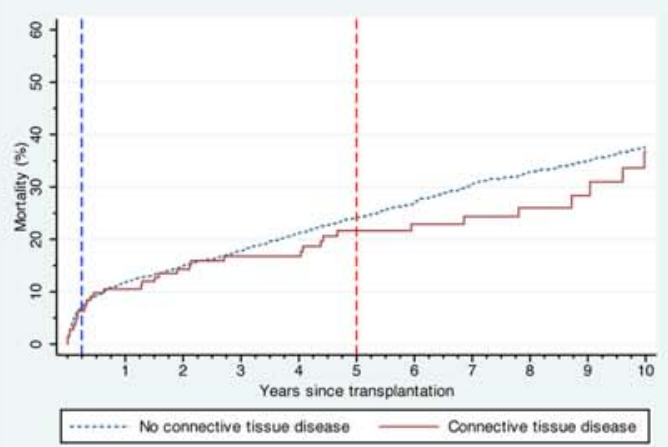

Diabetes Mellitus

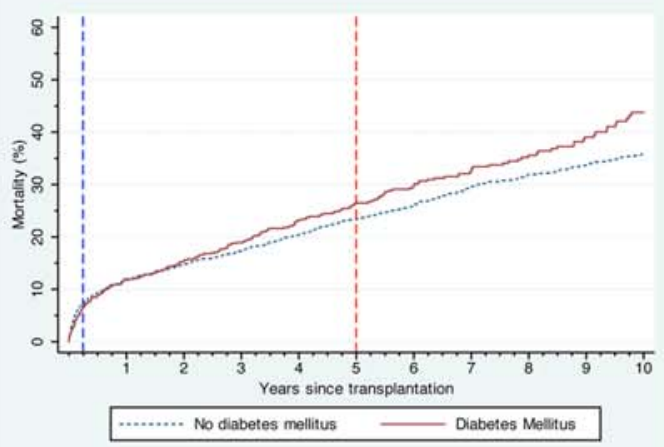

Chronic pulmonary disease

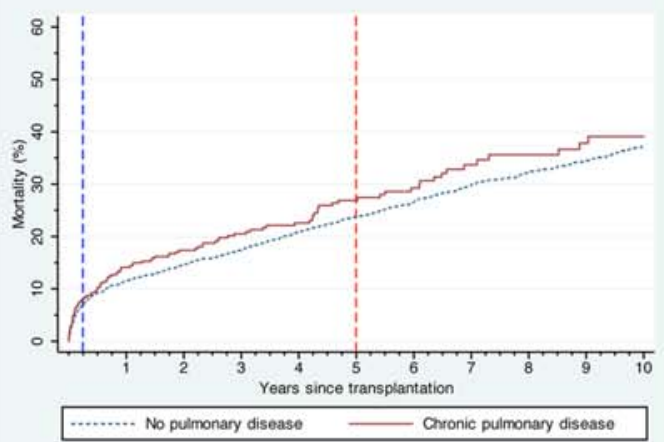

Congestive cardiac failure

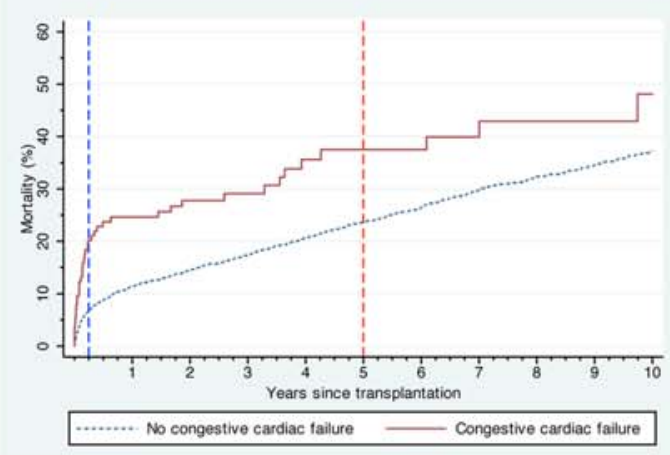

Dementia

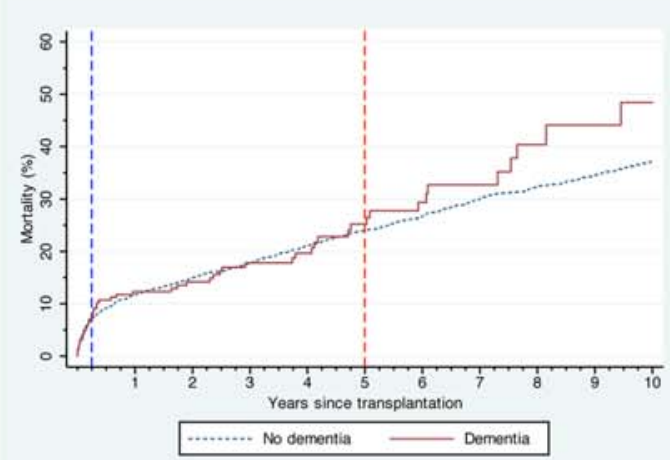

Non-hepatic malignancy

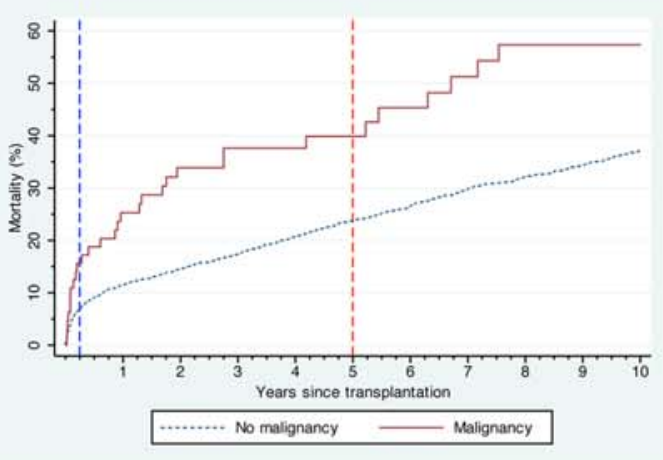

Chronic renal disease

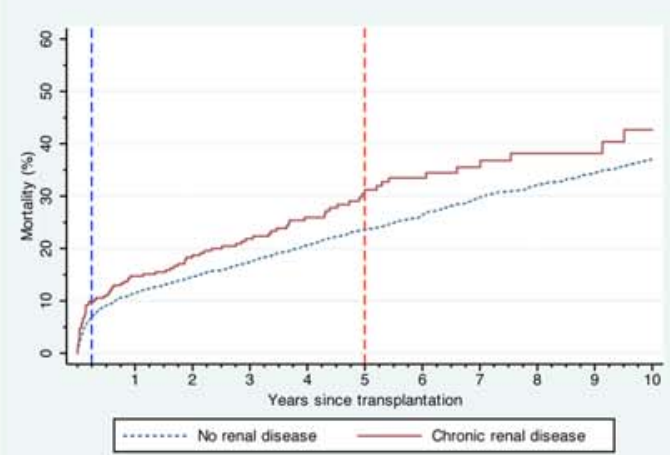

Figure 1 Kaplan-Meier post-transplant mortality curves of liver transplant recipients with and without comorbidity. 


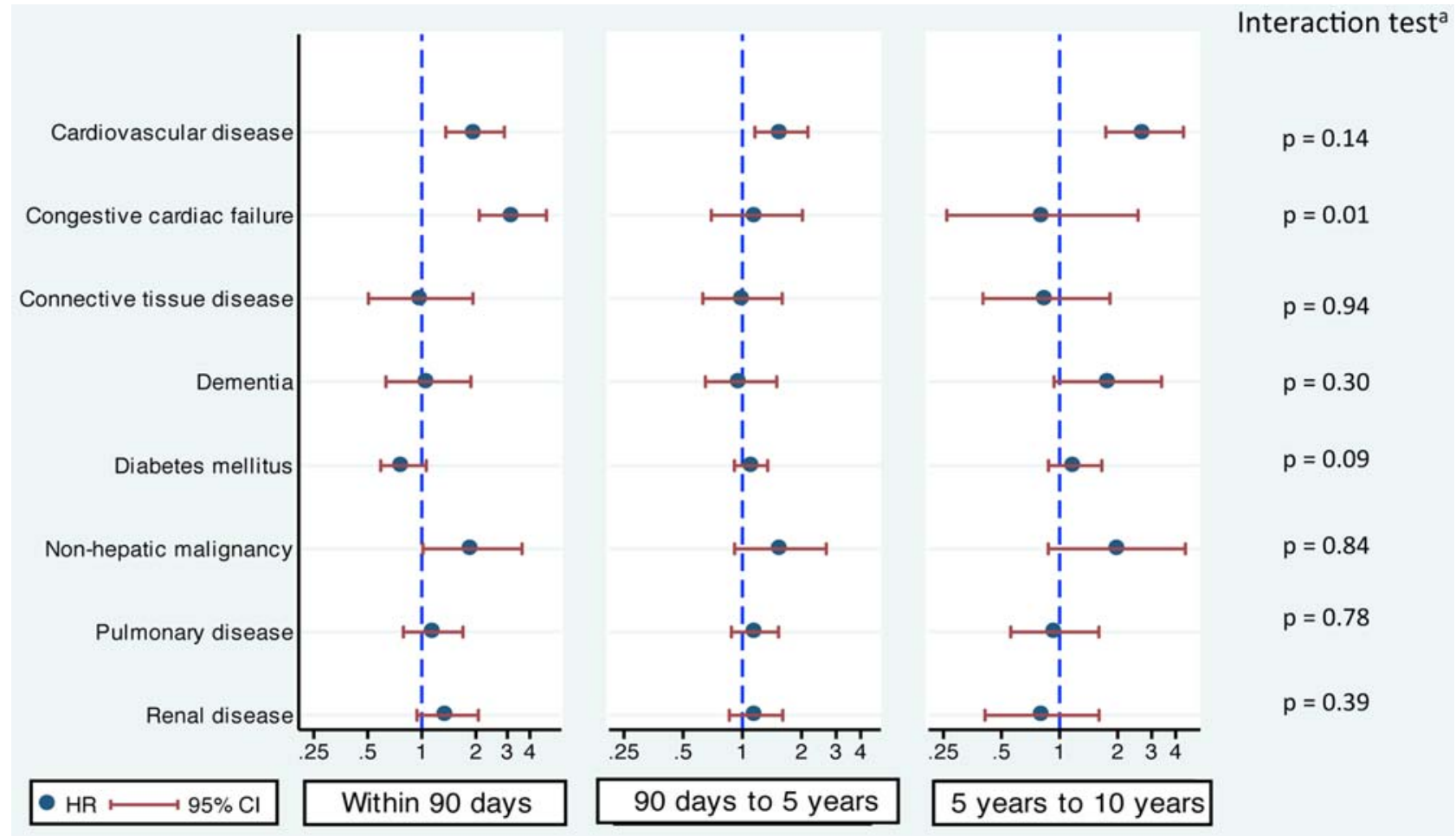

Figure 2 Adjusted HRs and their 95\% Cls of each comorbidity on patient mortality in the three time periods after liver transplantation (within 90 days, 90 days -5 years and 5-10 years). ${ }^{a}$ Interaction test compares HRs of the three time periods of survival after liver transplantation.

$1.5 \mathrm{mg} / \mathrm{dL}$ or greater as their definition. ${ }^{13}$ The differences in prevalence may also be due to coding methods or the patient selection criteria of the transplant programmes. We identified congestive cardiac failure as a comorbidity in $3 \%$ of our cohort, whereas it was particularly rare in the US $(0.5 \%)$ and Italian cohort $(0 \%)$. Our comorbidity coding scheme defines congestive cardiac failure as right or left ventricular failure (ICD-10 code I50), cardiomyopathy (I42, I43), cardiomegaly (I51.7), hypertensive heart failure (I11, I13) and ischaemic cardiomyopathy (I25.5). Prevalence of a history of non-hepatic malignancy in our cohort was $1.7 \%$, comparable to the US study (3\%), but much lower than in the Italian study (14\%).

In our analysis, cardiovascular disease was found to be a risk factor for mortality in all time periods after liver transplantation. Similarly, the US study found that coronary disease was a significant risk factor for posttransplant mortality $(\mathrm{HR}=2.3) .{ }^{13}$ Not only have we confirmed the increased risk of death in these patients, we also observed that the impact is more prominent in later years $(\mathrm{HR}=2.8)$. This suggests that liver transplant recipients with cardiovascular disease should have more intensive investigation and management of cardiovascular complications in the long term after transplantation.

A previous study did not find evidence to suggest that congestive cardiac failure increased the risk of death. ${ }^{13}$ However, it was a small study of 710 liver transplant recipients, only 3 of whom had congestive cardiac failure. In contrast, our study found that congestive cardiac failure was associated with higher mortality but only in the early post-transplant period. This short-term effect may be linked to the haemodynamically stressful effect of the operation, while the cardiac condition can be effectively controlled with medication in the long term.

Despite a relatively low prevalence of a history of nonhepatic malignancy in this liver transplant cohort $(1.7 \%$, 64 patients), we found that this comorbid condition was associated with an increased risk of death across all time periods, albeit only statistically significant in the first 90 days. Further review revealed that the most common prior malignancies were colorectal cancer (13 patients), cancer of unknown primary (12 patients) and leukaemia/lymphoma (11 patients) with a total of 27 patients having abdominal cancer. There were 30 deaths in the 64 patients with non-hepatic malignancy (10 patients in the first 90 days, 20 patients after 90 days). All the causes of death of the 10 patients who died within 90 days were not cancer-related (sepsis in 7 patients and multiple organ failure in 3 patients). Prior surgery associated with previous abdominal cancer treatment may have been responsible for the observed increased risk of early postoperative death in these patients. ${ }^{34}$ Additionally, these early deaths may have been indirectly related to the increased risk of sepsis arising from immunosuppression from prior cancer treatment. In this context, it is also important to note that among the patients with non- 
hepatic malignancy who died after 90 days, 14 of the 20 patients $(70 \%)$ had a malignancy coded as a cause of death.

There is conflicting evidence about whether diabetes is a risk factor for death after liver transplantation. Some studies did show a significant impact, ${ }^{10} 1335$ while others did not. ${ }^{34}{ }^{36}$ We found no evidence that diabetes had an impact on either short-term or long-term survival. This is most likely explained by the intensive screening for cardiovascular complications in diabetic liver transplant candidates in our cohort. It is an important observation that with such screening and careful patient selection as well as active diabetes management after transplantation, recipients with diabetes have outcomes comparable to recipients without diabetes. The absence of an impact of a diagnosis of diabetes on outcome after transplantation may also be because the multivariable Cox regression models were adjusted for cardiovascular disease, which is the main cause of post-transplant death in diabetic cases.

The prevalence of most of the comorbidities included in this study has increased over time. The prevalence of diabetes in liver transplant recipients almost doubled over 10 years from $17.4 \%$ before 2000 to $30.0 \%$ after 2010. This increase may be the results of improved coding of administrative data in the $\mathrm{UK},{ }^{37}$ the increased incidence of diabetes in the general population ${ }^{38}$ or the increase in prevalence of aetiologies of primary liver disease related to diabetes, including hepatitis C cirrhosis and non-alcoholic fatty liver disease. ${ }^{39} 40$ The percentage of recipients with at least one comorbidity has also increased, reflecting a willingness to list higher risk patients or patients with comorbidities for liver transplantation.

A strength of our study is that we could use a national clinical audit linked to administrative hospital data allowing us to study a wide range of comorbidities in a large number of patients. The administrative data provided information about comorbidity. In other studies of comorbidity in liver transplantation, comorbidities were identified through retrospective clinical chart review. ${ }^{13-16}$ Chart review is cumbersome as well as time and resource consuming. The number of patients included in such studies is often small. Comorbidities in liver transplant recipients are not that common, and a large sample size is required to study their effects on survival.

A number of limitations should be recognised. First, the administrative information recorded in the HES database was not originally designed for research purposes. It is possible that we have underestimated the prevalence of comorbidities because of incomplete coding, especially in earlier years. However, there is growing evidence that administrative data can be a reliable resource for clinical research. ${ }^{41-44}$ A key factor in improving its value for research is the development and validation of strategies to identify patients with particular conditions. Our study is an example of such work. Coding practice has improved and the overall coding of the primary diagnosis in the HES database has been shown to be accurate in $96 \%$ of the cases in recent years. $^{28}{ }^{37}$ Also, our comorbidity coding scheme used broad disease categories to limit the impact of coding error. Second, we found that $13 \%$ of the patients had two or more comorbidities. We explored the impact of individual comorbidities, but there was limited statistical power to investigate potential interaction effects of multiple comorbidities on outcome. Third, we acknowledge that by modifying an existing comorbidity index we may have missed other health problems that may have prognostic implications but were not included in the original index (eg, hypertension). However, the Charlson Comorbidity Index is a validated and extensively used comorbidity tool, which was constructed to capture most of the important comorbid conditions. Fourth, it is not always possible to distinguish liver-related conditions from true comorbidities. For example, volume overload, which is not an uncommon condition in patients with cirrhosis, may lead to symptoms mimicking congestive cardiac failure. Fifth, the grouping of comorbidities in the cardiovascular disease category is also a potential limitation because the observed effects on outcome cannot be attributed to an individual comorbidity. Effects of comorbidities that are in the same spectrum of diseases are likely to be in the same direction, but may not be of similar magnitude. Finally, our study was not designed to assess the impact of morbidity linked to or caused by liver diseases as a result of how comorbidity was defined.

\section{Implications}

Our ICD-10 coding scheme to extract comorbidity information relevant for liver transplantation from linked administrative data can contribute to other aspects of liver transplant research and service evaluation. For example, it can be included in prognostic models used for risk adjustment when outcomes after liver transplantation between centres are compared. Comorbidity information should be used in conjunction with all other available risk factors to improve the selection and allocation process of liver transplantation.

Congestive cardiac failure has a significant effect only on short-term mortality, while cardiovascular disease is associated with increased short-term, mid-term and longterm mortality. Non-hepatic malignancy is associated with higher mortality over the whole follow-up period. It is important that analysis methods allow for potential time-varying effects over a long follow-up period.

Liver transplant patients should be managed according to their specific comorbidities and follow-up periods. Our data suggest that with careful screening and management, patients with diabetes and those with renal and pulmonary disease can be safely transplanted with comparable outcomes, while patients with cardiovascular disease have higher risk and should be carefully investigated and managed for its complications in the long term. 
Acknowledgements The authors would like to thank all liver transplant centres for providing data to the UK Liver Transplant Audit. They would also like to thank all those involved in collecting and handling liver transplant data at the National Health Service Blood and Transplant. The UK Liver Transplant Audit is supported by the NHS National Specialised Commissioning Group/ NHS England. HES data have been reused with the permission of The Health and Social Care Information Centre. CT is supported by the Faculty Scholarship from Faculty of Medicine Siriraj Hospital, Mahidol University, Bangkok, Thailand, and is a recipient of the 'Raymond and Beverly Sackler Studentship' from School of Clinical Medicine, University of Cambridge, Cambridge, UK.

Contributors CT participated in research design, performed the data and statistical analysis and drafted the manuscript. SCC participated in research design and statistical analysis. JvdM participated in research design and drafting the manuscript. All authors contributed to the interpretation of the results, commented on drafts and approved the final manuscript.

Funding This research received no specific grant from any funding agency in the public, commercial or not-for-profit sectors.

\section{Competing interests None declared.}

Ethics approval This study is exempt UK National Research Ethics Service approval as it involved analysis of existing databases of anonymised data for service evaluation. Approval for the use of Hospital Episode Statistics data was obtained as the standard Hospital Episodes Statistics approval process.

Provenance and peer review Not commissioned; externally peer reviewed.

Data sharing statement The technical appendix and statistical code are available from the corresponding author.

Open Access This is an Open Access article distributed in accordance with the Creative Commons Attribution Non Commercial (CC BY-NC 4.0) license, which permits others to distribute, remix, adapt, build upon this work noncommercially, and license their derivative works on different terms, provided the original work is properly cited and the use is non-commercial. See: http:// creativecommons.org/licenses/by-nc/4.0/

\section{REFERENCES}

1. de Groot V, Beckerman H, Lankhorst GJ, et al. How to measure comorbidity. A critical review of available methods. J Clin Epidemiol 2003;56:221-9.

2. Linn BS, Linn MW, Gurel L. Cumulative illness rating scale. J Am Geriatr Soc 1968;16:622-6.

3. Kaplan $\mathrm{MH}$, Feinstein AR. The importance of classifying initial co-morbidity in evaluating the outcome of diabetes mellitus. J Chronic Dis 1974;27:387-404.

4. Greenfield S, Apolone G, McNeil BJ, et al. The importance of co-existent disease in the occurrence of postoperative complications and one-year recovery in patients undergoing total hip replacement. Comorbidity and outcomes after hip replacement. Med Care 1993;31:141-54.

5. Parkerson GR Jr, Broadhead WE, Tse CK. The Duke Severity of Illness Checklist (DUSOI) for measurement of severity and comorbidity. J Clin Epidemiol 1993;46:379-93.

6. Mulrow CD, Gerety MB, Cornell JE, et al. The relationship between disease and function and perceived health in very frail elders. $J \mathrm{Am}$ Geriatr Soc 1994;42:374-80.

7. Nair S, Verma S, Thuluvath PJ. Pretransplant renal function predicts survival in patients undergoing orthotopic liver transplantation. Hepatology 2002;35:1179-85.

8. Yoo HY, Thuluvath PJ. The effect of insulin-dependent diabetes mellitus on outcome of liver transplantation. Transplantation 2002;74:1007-12.

9. Plotkin JS, Scott VL, Pinna A, et al. Morbidity and mortality in patients with coronary artery disease undergoing orthotopic liver transplantation. Liver Transpl Surg 1996;2:426-30.

10. Samuelson AL, Lee M, Kamal A, et al. Diabetes mellitus increases the risk of mortality following liver transplantation independent of MELD score. Dig Dis Sci 2010;55:2089-94.

11. Wray C, Scovotti JC, Tobis J, et al. Liver transplantation outcome in patients with angiographically proven coronary artery disease: a multi-institutional study. Am J Transplant 2013;13:184-91.
12. Charlson ME, Pompei $\mathrm{P}$, Ales $\mathrm{KL}$, et al. A new method of classifying prognostic comorbidity in longitudinal studies: development and validation. J Chron Dis 1987;40:373-83

13. Volk ML, Hernandez JC, Lok AS, et al. Modified Charlson comorbidity index for predicting survival after liver transplantation. Liver Transpl 2007;13:1515-20.

14. Grosso G, di Francesco F, Vizzini G, et al. The Charlson comorbidity index as a predictor of outcomes in liver transplantation: single-center experience. Transplant Proc 2012;44:1298-302.

15. Wasilewicz M, Raszeja-Wyszomirska J, Wunsch E, et al. Modified Charlson Comorbidity Index in predicting early mortality after liver transplantation. Transplant Proc 2009;41:3117-18.

16. Basile-Filho A, Nicolini EA, Auxiliadora-Martins $\mathrm{M}$, et al. Comparison of acute physiology and chronic health evaluation II death risk, Child-Pugh, Charlson, and model for end-stage liver disease indexes to predict early mortality after liver transplantation. Transplant Proc 2011;43:1660-4.

17. Bird SM, Calne RY, Sharples LD. Analyse transplant outcomes in distinct epochs of follow-up. Lancet 2006;367:1816.

18. Dawwas MF, Gimson AE, Lewsey JD, et al. Survival after liver transplantation in the United Kingdom and Ireland compared with the United States. Gut 2007;56:1606-13.

19. Dowsley TF, Bayne DB, Langnas AN, et al. Diastolic dysfunction in patients with end-stage liver disease is associated with development of heart failure early after liver transplantation. Transplantation 2012;94:646-51.

20. Armitage JN, van der Meulen $\mathrm{JH}$. Identifying co-morbidity in surgical patients using administrative data with the Royal College of Surgeons Charlson Score. Br J Surg 2010;97:772-81.

21. Jameson SS, Baker PN, Charman SC, et al. The effect of aspirin and low-molecular-weight heparin on venous thromboembolism after knee replacement: a non-randomised comparison using National Joint Registry Data. J Bone Joint Surg Br 2012;94:914-18.

22. Jeevan $\mathrm{R}$, Cromwell DA, Trivella $\mathrm{M}$, et al. Reoperation rates after breast conserving surgery for breast cancer among women in England: retrospective study of hospital episode statistics. BMJ 2012;345:e4505.

23. Johal A, Mitchell D, Lees T, et al. Use of Hospital Episode Statistics to investigate abdominal aortic aneurysm surgery. Br J Surg 2012;99:66-72

24. van der Meulen JH, Lewsey JD, Dawwas MF, et al. Adult orthotopic liver transplantation in the United Kingdom and Ireland between 1994 and 2005. Transplantation 2007;84:572-9.

25. Hospital Episode Statistics. http://www.hscic.gov.uk/hes (accessed February 2014).

26. World Health Organization. International statistical classification of diseases and related health problems. 10th revision edn. Geneva: World Health Organization, 1992

27. NHS Classifications Service. OPCS classification of interventions and procedures: version 4.4 (April 2007). London: TSO, 2007.

28. Tovikkai C, Charman SC, Praseedom RK, et al. Linkage of a national clinical liver transplant database with administrative hospital data: methods and validation. Transplantation 2014;98:341-7.

29. Charman SC, Copley L, Tovikkai C, et al. UK Liver Transplant Audit in patients who received a liver transplant between 1st March 1994 and 31st March 2012. Annual Report to the National Specialist Commissioning Advisory Group: The Royal College of Surgeons of England, 2012. http://www.rcseng.ac.uk/surgeons/research/ surgical-research/docs/liver-transplant-audit-report-2012/view (accessed Aug 2014)

30. Neuberger J, Gimson A, Davies M, et al. Selection of patients for liver transplantation and allocation of donated livers in the UK. Gut 2008;57:252-7

31. Hosmer DW, Lemeshow S. Applied survival analysis: regression modeling of time to event data. New York: Wiley, 1999:225-30.

32. White IR, Royston P, Wood AM. Multiple imputation using chained equations: issues and guidance for practice. Stat Med 2011;30:377-99.

33. Marshall A, Altman DG, Holder RL, et al. Combining estimates of interest in prognostic modelling studies after multiple imputation: current practice and guidelines. BMC Med Res Methodol 2009;9:57.

34. Rana A, Hardy MA, Halazun KJ, et al. Survival outcomes following liver transplantation (SOFT) score: a novel method to predict patient survival following liver transplantation. Am J Transplant 2008;8:2537-46.

35. Aloia TA, Knight R, Gaber AO, et al. Analysis of liver transplant outcomes for United Network for Organ Sharing recipients 60 years old or older identifies multiple model for end-stage liver diseaseindependent prognostic factors. Liver Transp/ 2010;16:950-9.

36. Nair S, Vanatta JM, Arteh J, et al. Effects of obesity, diabetes, and prior abdominal surgery on resource utilization in liver transplantation: a single-center study. Liver Transp/ 2009;15:1519-24. 
37. Burns EM, Rigby E, Mamidanna R, et al. Systematic review of discharge coding accuracy. J Public Health (Oxf) 2012;34:138-48.

38. Holden SH, Barnett AH, Peters JR, et al. The incidence of type 2 diabetes in the United Kingdom from 1991 to 2010. Diabetes Obes Metab 2013;15:844-52.

39. Mason AL, Lau JY, Hoang N, et al. Association of diabetes mellitus and chronic hepatitis C virus infection. Hepatology 1999;29:328-33.

40. Adam R, Karam V, Delvart V, et al. Evolution of indications and results of liver transplantation in Europe. A report from the European Liver Transplant Registry (ELTR). J Hepatol 2012;57:675-88.

41. Slavin JP, Deakin M, Wilson R. Surgical research and activity analysis using Hospital Episode Statistics. Ann R Coll Surg Engl 2012:94:537-8
42. Smith AJ, Dieppe P, Porter M, et al. Risk of cancer in first seven years after metal-on-metal hip replacement compared with other bearings and general population: linkage study between the National Joint Registry of England and Wales and hospital episode statistics. BMJ 2012;344:e2383.

43. Karthikesalingam A, Holt PJ, Patterson BO, et al. Elective open suprarenal aneurysm repair in England from 2000 to 2010 an observational study of hospital episode statistics. PLOS ONE 2013;8: e64163.

44. Sinha S, Hofman D, Stoker DL, et al. Epidemiological study of provision of cholecystectomy in England from 2000 to 2009: retrospective analysis of Hospital Episode Statistics. Surg Endosc 2013:27:162-75. 